\title{
Erratum to: Hyper-gourd theory: solving simultaneously the mysteries in particle physics, biology, oncology, neurology, economics, and cosmology
}

\author{
Ken Naitoh
}

Published online: 19 January 2013

(C) ISAROB 2013

Erratum to: Artif Life Robotics (2012) 17(2):275-286 DOI 10.1007/s10015-012-0056-y

Among the quasi-stable size ratios of $\varepsilon=r_{d i} / r_{d j}[i, j=$ $1,2, i \neq j]$ for two spheroid-like parcels connected, i.e., super-magic numbers in Table 1 , the ratios related to the convection force for $m \neq 1$ and $\varepsilon \neq 1$ appear with the statistic indeterminacy of the contact position of two parcels, whereas the other ratios can exist even when the statistic indeterminacy effect due to small number of subparticles inside a parcel is absent.

For details, the relation between dimensionless deformation rate $\gamma_{k}\left(\equiv a_{k} / b_{k}[k=1,2]\right)$ of each parcel dependent on dimensionless time and the size ratio of the two parcels $\varepsilon=r_{d i} / r_{d j}[i, j=1,2, i \neq j]$, i.e., Eq. 2, should be described as

$$
\begin{aligned}
\frac{\mathrm{d}^{2}}{\mathrm{~d} \bar{t}_{i}^{2}} \gamma_{i}= & \left\{m_{c i}\left(\frac{\mathrm{d}}{\mathrm{d} \bar{t}_{i}} \gamma_{i}\right)^{2}+m_{c j}\left(\frac{\mathrm{d}}{\mathrm{d} \bar{t}_{j}} \gamma_{j}\right)^{2}+m_{s i} \gamma_{i}^{\frac{5}{3}-\frac{2}{3} m}+m_{s j} \gamma_{j}^{\frac{5}{3}-\frac{2}{3} m}\right\} / \\
& \text { Det }+\delta_{s t} \quad[\text { for } i=1,2 . j=1,2 . i \neq j]
\end{aligned}
$$

with

The online version of the original article can be found under doi:10.1007/s10015-012-0056-y.

K. Naitoh $(\bowtie)$

Faculty of Science and Engineering, Waseda University,

3-4-1 Ookubo, Shinjuku, Tokyo 169-8555, Japan

e-mail:k-naito@waseda.jp

URL: http://www.k-naito.mech.waseda.ac.jp/;

https://www.wnp7.waseda.jp/Rdb/app/ip/ipi0211.html?

lang_kbn=0\&kensaku_no=4181

$$
\begin{aligned}
& m_{c i}=\left[\left(-\varepsilon-\varepsilon^{4}+\frac{2}{3} \varepsilon E_{0 j} \gamma_{j}^{-1 / 3}\right) B_{0 i}+\frac{2}{9} \varepsilon^{4-2 \Delta m} E_{0 i} \gamma_{i}^{-4 / 3}\right] \\
& m_{c j}=\left[\frac{2}{3} \varepsilon^{2+m} E_{0 i} \gamma_{j}^{-1 / 3} B_{0 j}-\frac{2}{9} \varepsilon^{2+m} E_{0 i} \gamma_{j}^{-4 / 3}\right] \\
& m_{s i}=\left(-\varepsilon-\varepsilon^{4}+\frac{2}{3} \varepsilon E_{0 j} \gamma_{j}^{-1 / 3}\right) C_{0 i} \\
& m_{s j}=\frac{2}{3} \varepsilon^{2+m} E_{0 i} \gamma_{j}^{-1 / 3} C_{0 j} \\
& \text { Det }=-\frac{2}{\varepsilon}-\varepsilon^{4}+\frac{2}{3} \varepsilon^{4} E_{0 i} \gamma_{i}^{-1 / 3}+\frac{2}{3} \varepsilon E_{0 j} \gamma_{j}^{-1 / 3} \\
& B_{0 k}=\frac{1}{3 \gamma_{k}} \frac{\gamma_{k}^{2}-2}{\gamma_{k}^{2}-1 / 2}, \\
& C_{0 k}=\frac{3}{8} \frac{2 \gamma_{k}^{2 m}-1 / \gamma_{k}^{m}-\gamma_{k}^{m}}{\gamma_{k}^{2}-1 / 2}, \text { and } \\
& E_{0 k}=3 \frac{\gamma_{k}^{7 / 3}}{\gamma_{k}^{2}-1 / 2} \quad[\text { for } k=1,2]
\end{aligned}
$$

where the parameter $\delta_{s t}$ and $\Delta m$ denote the random force due to small number of sub-particles inside a parcel and the indeterminacy effect (stochastic variation) of the contact surface position between two parcels connected, respectively. When we assume the relation of $\Delta m=(1-m) / 2$, Table 1 can be obtained. 